\title{
Brainstem cranial nerve diffusion changes in Wernicke encephalopathy secondary to necrotizing pancreatitis
}

Serpil Kurtcan, Alpay Alkan, Sinem Aydin, Umit Tuzun, Huseyin Toprak

Department of Radiology, Bezmialem Vakif University, Istanbul, Turkey

Submitted: 17 May 2016

Accepted: 18 May 2016

Arch Med Sci Civil Dis 2016; 1: e45-e50

DOI: 10.5114/amscd.2016.60255

Copyright $\odot 2016$ Termedia \& Banach

Wernicke encephalopathy (WE) is a neurologic disorder related to the deficiency of thiamine (vitamin $B_{1}$ ). Thiamine has an important role as a coenzyme of transketolase, $\alpha$-ketoglutarate dehydrogenase and pyruvate dehydrogenase complex. Thiamine deficiency often results in an osmotic gradient disorder which leads to intracellular and extracellular edema [1].

Wernicke encephalopathy commonly occurs due to chronic alcohol abuse. However, some clinical conditions such as gastrointestinal surgery, prolonged vomiting, dietary unbalance, hyperemesis gravidarum and anorexia nervosa may cause WE [2]. The classical symptoms are usually characterized by a clinical triad involving changes in consciousness, ocular dysfunction, and ataxia. Early diagnosis and treatment are crucial for prognosis.

Typical magnetic resonance imaging (MRI) features include symmetric hyperintensity in the mamillary bodies, medial thalami, tectal plate, and periaqueductal area on T2 weighted imaging [3]. Also, there are some studies reporting atypical MRI findings including signal intensity alterations in different brain areas such as the cerebellum, cranial nerve nuclei, tegmentum of the lower pons, cerebellar dentate nuclei, caudate nuclei, splenium of corpus callosum, and cerebral cortex [4]. However, as far as we know, there is no study showing diffusion changes of cranial nerve nuclei involvement.

A 36-year-old male patient had been hospitalized with the diagnosis of necrotizing pancreatitis. He had no history of alcohol abuse. Computed tomography (CT) showed acute pancreatitis and accompanying necrosis. Total parenteral nutrition and antibiotic therapy were started for the patient. For the last 2 days, he had difficulty in walking, drowsiness and ocular symptoms and deterioration in the level of consciousness. On the neurologic examination, pupils were isochoric and pupillary light reflexes were positive. Papilledema was not detected, but there was horizontal divergent gazing bilaterally. Spontaneous movements of the extremities and deep tendon reflexes were positive. Kidney functions, serum glucose and total protein values were normal. Serum concentrations of alkaline phosphatase (ALP), lactate dehydrogenase (LDH), $\gamma$-glumatyl transferase (GGT) and amylase were mildly elevated. $\mathrm{Ca}, \mathrm{Na}$ and $\mathrm{Cl}$ levels were mildly decreased. Serum thiamine concentration was $15 \mathrm{ng} /$ $\mathrm{ml}$ (norm: 20-50). On the diffusion-weighted imaging (DWI), symmetric hyperintensities were detected in mammillary bodies, medial thalami, the periaqueductal area and in the prepositus hypoglossal nuclei at the medulla oblongata with isointensity on the ADC map (Figure 1). With

\author{
Corresponding author: \\ Serpil Kurtcan \\ Department of Radiology \\ Bezmialem Vakif University \\ 42017 Istanbul, Turkey \\ Phone: +9002124531700 \\ E-mail: s.kurtcan@hotmail. \\ com
}




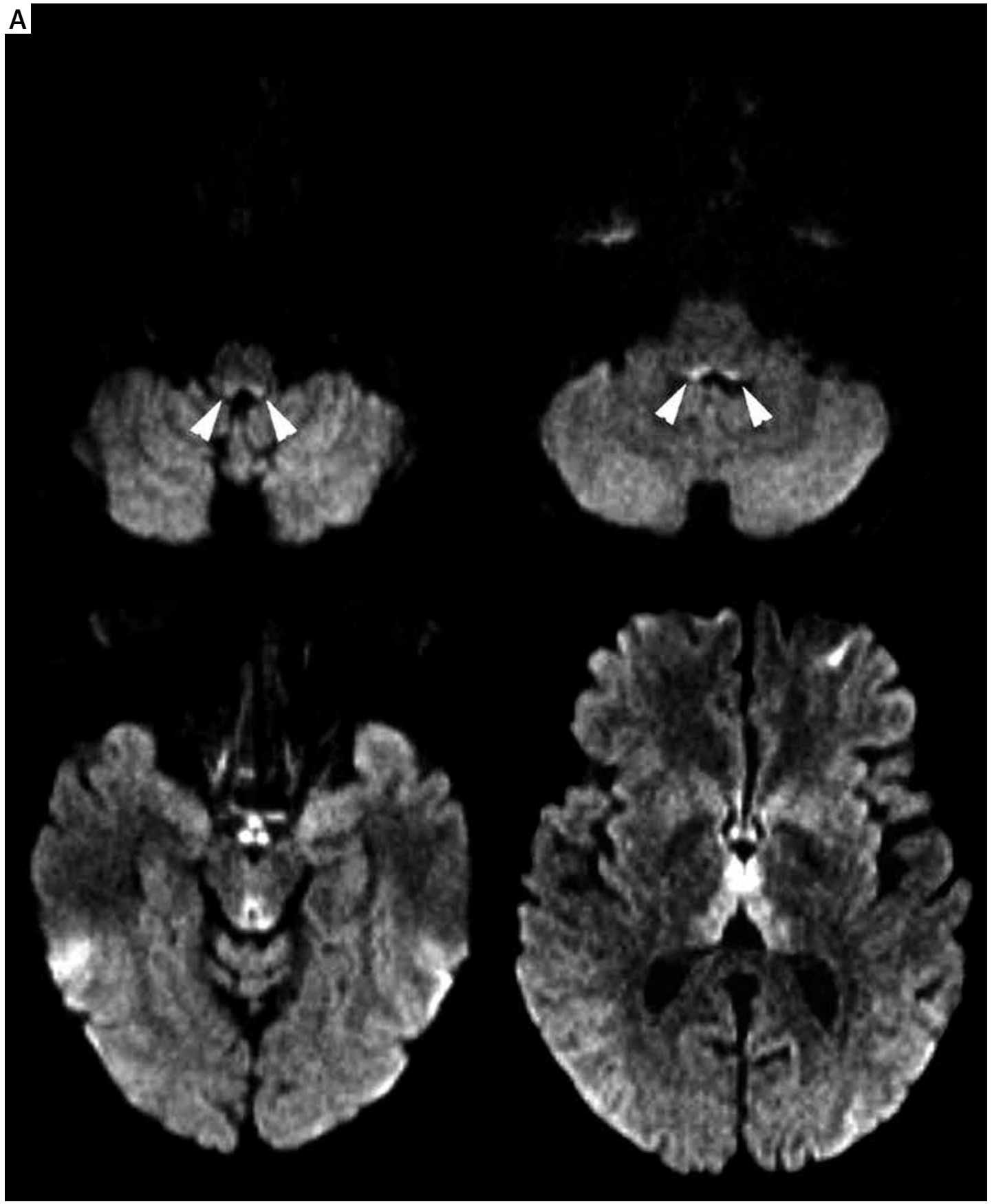

Figure 1. A - A 36-year-old male patient with WE. Initial diffusion-weighted images show high signal intensities in the bilateral median thalami, mamillary bodies, periaqueductal area and prepositus hypoglossal nuclei at the lower pons and medulla oblongata level (arrowheads)

thorough clinical assessment and according to the DWI findings, the patient was diagnosed with WE. Daily i.v. $100 \mathrm{mg}$ thiamine therapy was started. On the DWI after 1 week, there was mild regression in signal intensities in mammillary bodies, medial thalami and periaqueductal area and further regression in the prepositus hypoglossal nuclei on the medulla oblongata. Furthermore, newly developed lesions were detected in the left nucleus caudatus, bilateral putamen and cerebral cortex with low ADC values (Figure 2). During follow-up, the ocular dysfunction improved after thiamine supplementation although other symptoms persisted in the patient. Control DWI performed 3 weeks after the diagnosis showed that the signal intensity observed in the putamen persisted, but the hyperintensities in the cerebral cortex had regressed. Additionally, newly developed symmetric hyperintensities on the pontine nuclei were observed (Figure 3). While the treatment of the patient in the intensive care unit was ongoing, due to worsening of his clinical condition, the patient was intubat- 


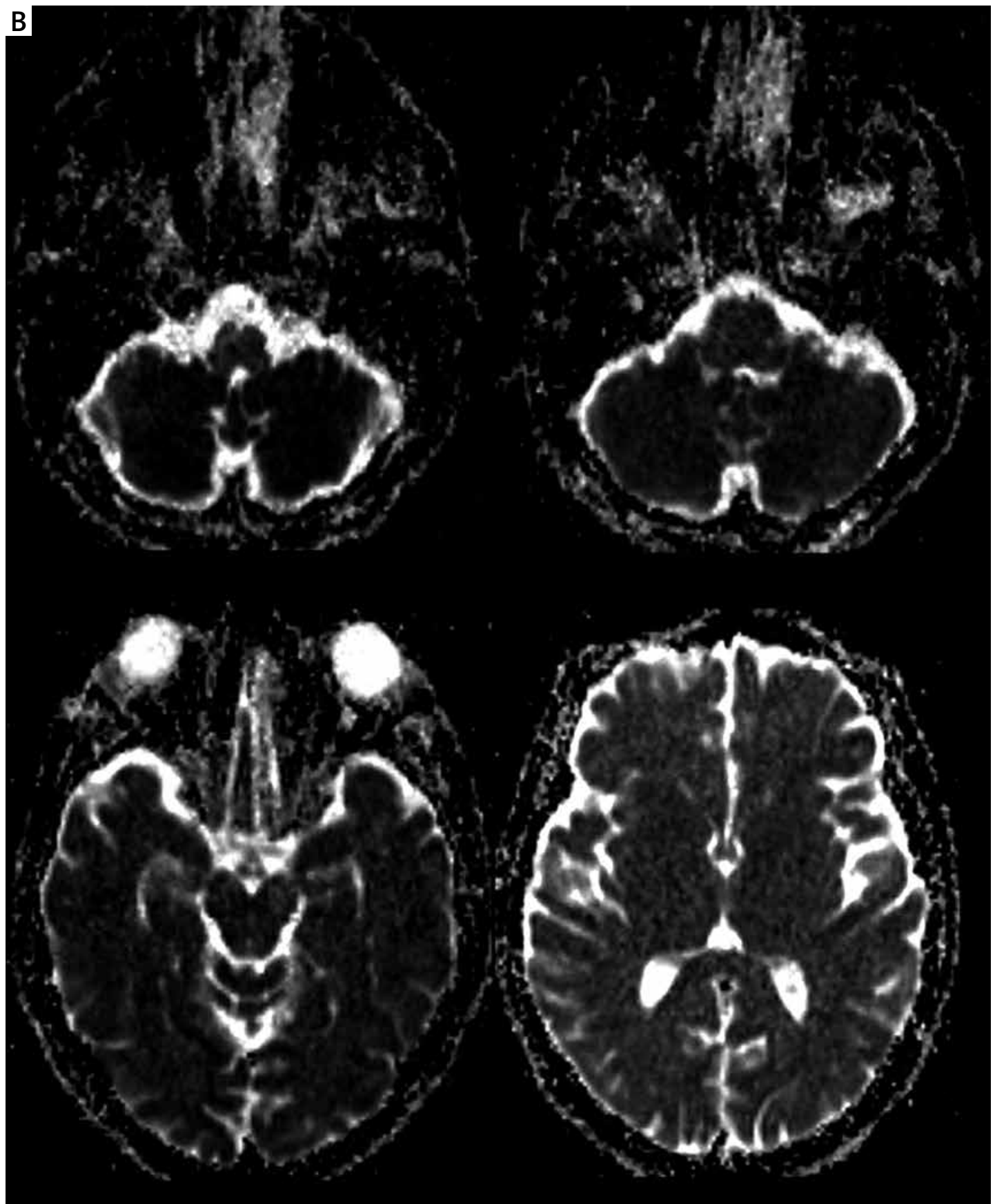

Figure 1. B - A 36-year-old male patient with WE. Initial ADC map images demonstrate isointensity in the bilateral median thalami, mamillary bodies, periaqueductal area and prepositus hypoglossal nuclei at the lower pons and medulla oblongata level

ed. However, he died two and a half months after hospitalization.

The daily thiamine requirement obtained by carbohydrate (dietary) intake for healthy individuals is $1-2 \mathrm{mg}$. The thiamine reserve in the body is about $30-50 \mathrm{mg}$. This reserve in the body is only sufficient for 4-6 weeks under conditions leading to thiamine deficiency.

The pathogenesis of WE due to thiamine deficiency is not certainly identified. Impaired glucose metabolism in Krebs and pentose phosphate cy- cles and increase in the intracellular concentration of glutamate because of the enzymatic inactivity are accepted to be responsible for WE. As a result, cellular homeostasis breaks down and glutamate is released to the extracellular region [5]. The increase in extracellular glutamate is known to be one of the primary causes of excitotoxic brain damage. In addition, increased extracellular glutamate results in axonal swelling and cytotoxic edema of glial cells, possibly contributing to diffusion abnormalities leading to necrosis. 


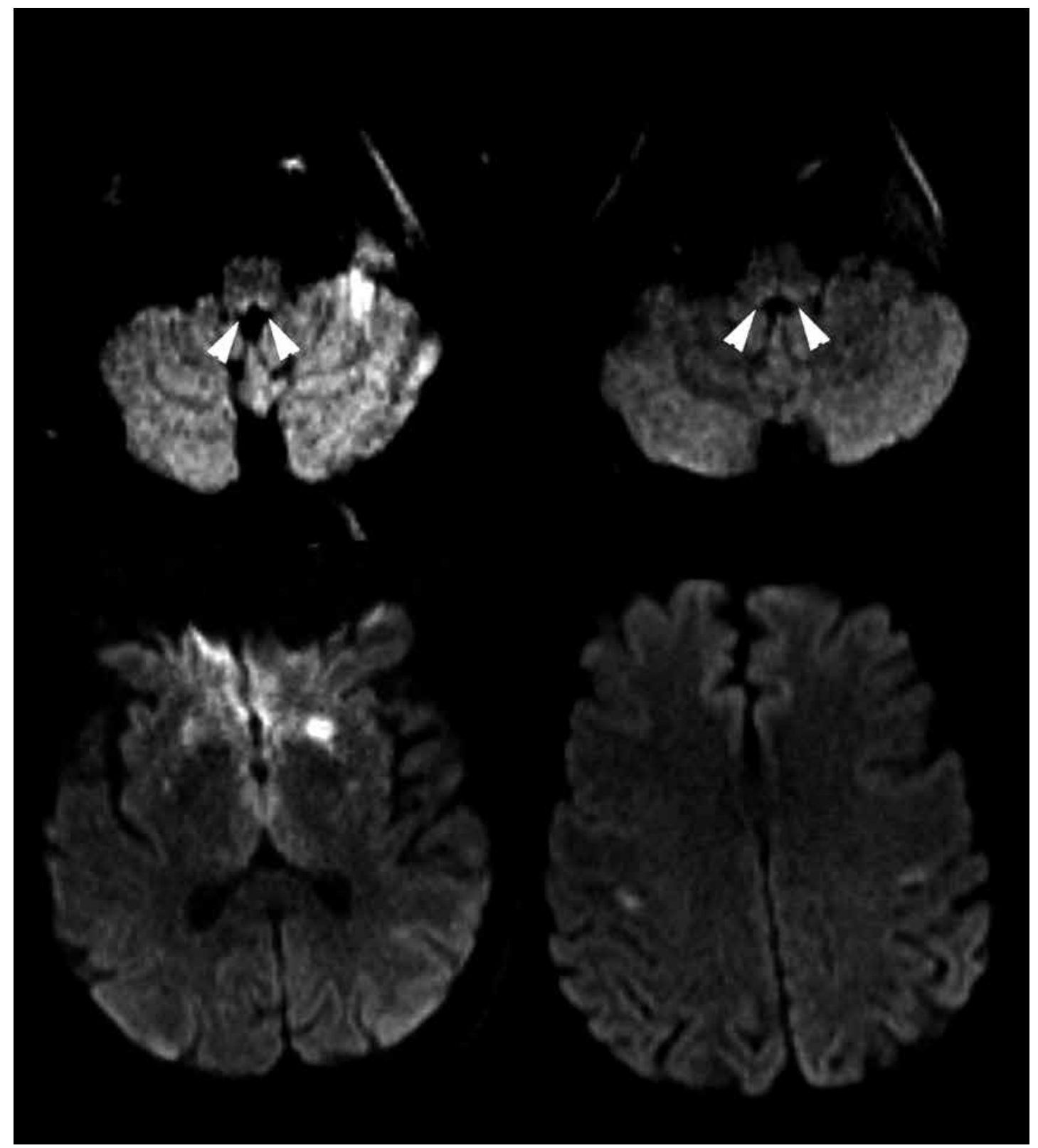

Figure 2. The follow-up DWI 1 week after thiamine therapy. Diffusion-weighted images show mild regression in signal intensities in medial thalami and prepositus hypoglossal nuclei at the lower pons and medulla oblongata level (arrowheads). There are newly developed lesions in the bilateral putamen and cerebral cortex on DWI

In recent studies, the involvement of prepositus hypoglossal nuclei at the medulla oblongata, the cerebral cortex and cranial nerve nuclei at the level of the lower pons on conventional MRI has been reported [6-8]. Ha et al. [8] identified dorsal medulla involvement on FLAIR images. They also reported that the medulla oblongata contains a great number of cranial nerve nuclei, and these cranial nerve nuclei could not be differentiated on FLAIR images. In our study, we identified symmetric involvement of the prepositus hypoglossal nuclei at the medulla oblongata on DWI as well as the other typical areas. We observed diffusion alterations in the cerebral cortex, basal ganglia and pontine nuclei. The DWI is a method which can detect alterations in the diffusion of water molecules and may show early ischemic changes in brain tissue.

Wernicke encephalopathy lesions can be observed as hyperintensities on DWI, with increased, low or normal ADC values. The hyperintense lesions detected together with low ADC are consistent with cytotoxic edema, whereas the increased ADC is consistent with vasogenic edema. Some studies have reported that the hyperintense lesions detected on DWI with a normal ADC value could result from T2 shine-through effects [9]. In our patient, DWI showed hyperintensity on the 


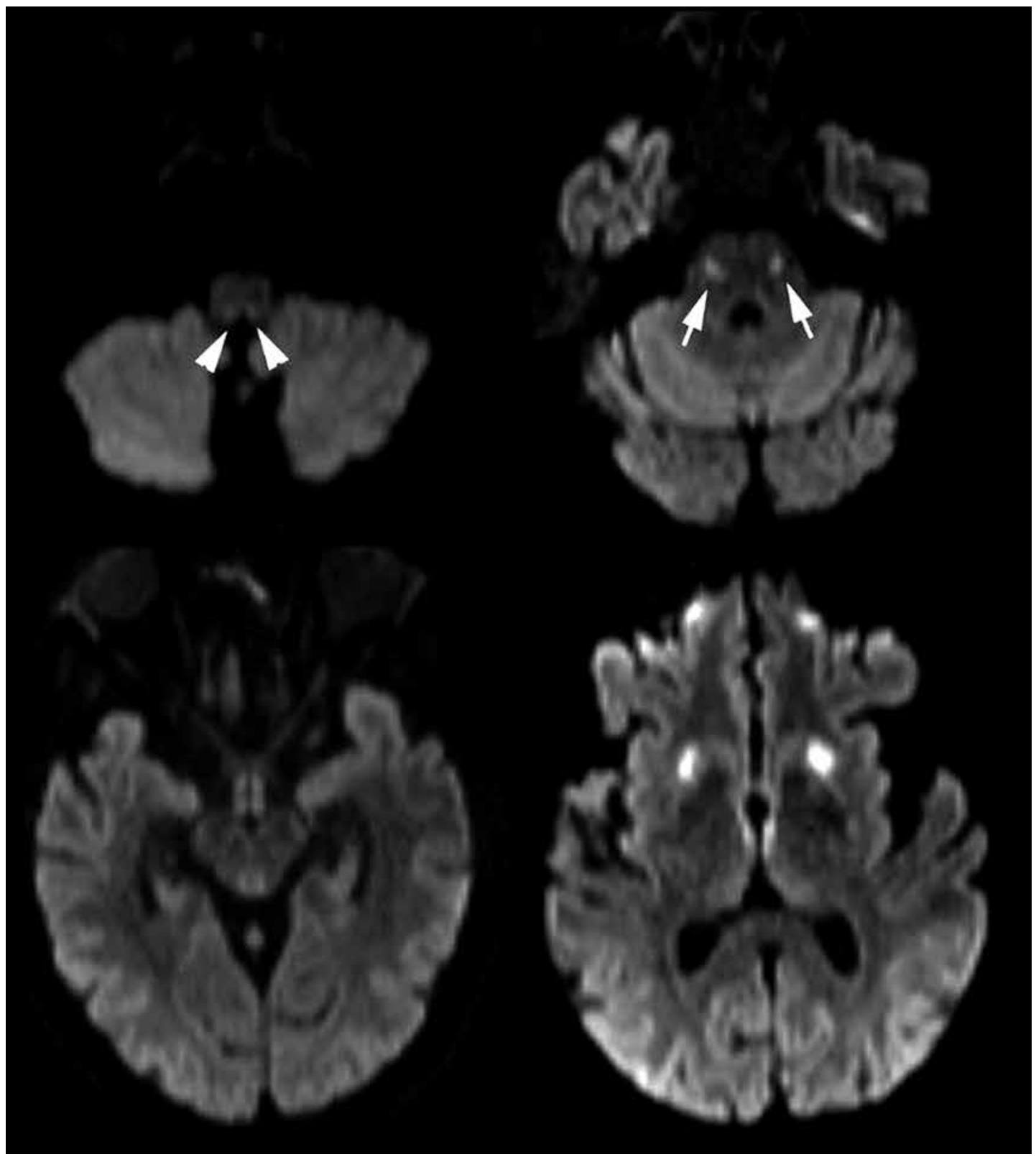

Figure 3. The follow-up DWI performed 3 weeks after the diagnosis. Diffusion-weighted image shows persisted signal intensities in the putamen. Newly developed symmetric high signal intensities on the pontine nucleus at the pons level on DWI were observed (arrows)

left nucleus caudatus, bilateral putamen and cerebral cortex with low ADC, which supports cytotoxic edema. Diffusion changes in the other regions, especially at the cranial nerve nuclei, may reflect the difference in the degree of cytotoxic edema.

In conclusion, the hyperintensities detected on DWI indicate the tissue at risk, but do not necessarily show irreversibility [5]. By means of early diagnosis and adequate treatment, abnormalities on DWI may also be reversible [10]. The DWI must be added to routine MRI sequences in diagnosis of the acute phase and follow-up period of WE to demonstrate cranial nerve involvement and to assess the response to therapy.

\section{Conflict of interest}

The authors declare no conflict of interest.

\section{References}

1. Schroth G, Wichmann W, Valavanis A. Blood-brain-barrier disruption in acute Wernicke encephalopathy: $M R$ findings. J Comput Assist Tomogr 1991; 15: 1059-61.

2. Chiossi G, Neri I, Cavazzuti M, Basso G, Facchinetti F. Hyperemesis gravidarum complicated by Wernicke encephalopathy: background, case report, and review of the literature. Obstet Gynecol Surv 2006; 61: 255-68.

3. Zuccoli G, Gallucci M, Capellades J, et al. Wernicke encephalopathy: MR findings at clinical presentation in 
twenty-six alcoholic and nonalcoholic patients. Am J Neuroradiol 2007; 28: 1328-31.

4. Zuccoli G, Motti L. Atypical Wernicke's encephalopathy showing lesions in the cranial nerve nuclei and cerebellum. J Neuroimaging 2008; 18: 194-7.

5. Chu K, Kang DW, Kim HJ, Lee YS, Park SH. Diffusion-weighted imaging abnormalities in Wernicke encephalopathy: reversible cytotoxic edema? Arch Neurol 2002; 59: 123-7.

6. Zuccoli G, Santa Cruz D, Bertolini M, et al. MR imaging findings in 56 patients with Wernicke encephalopathy: nonalcoholics may differ from alcoholics. Am J Neuroradiol 2009; 30: 171-6.

7. Bae SJ, Lee HK, Lee JH, Choi CG, Suh DC. Wernicke's encephalopathy: atypical manifestation at MR imaging. Am J Neuroradiol 2001; 22: 1480-2.

8. Ha ND, Weon YC, Jang JC, Kang BS, Choi SH. Spectrum of $M R$ imaging findings in Wernicke encephalopathy: are atypical areas of involvement only present in nonalcoholic patients? Am J Neuroradiol 2012; 33: 1398-402.

9. Doherty MJ, Watson NF, Uchino K, Hallam DK, Cramer SC. Diffusion abnormalities in patients with Wernicke encephalopathy. Neurology 2002; 58: 655-7.

10. Rugilo CA, Roca MC, Zurru MC, Gatto EM. Diffusion abnormalities and Wernicke encephalopathy. Neurology 2003; 60: 727-8. 